\title{
Carbon-ion radiotherapy in the treatment of radiation-induced second primary malignancies
}

\author{
Jing Yang ${ }^{1,2}$, Jing Gao ${ }^{1,2}$, Jiyi Hu ${ }^{1,2}$, Weixu Hu ${ }^{1,2}$, Xianxin Qiu ${ }^{1,2}$, Qingting Huang ${ }^{1,2}$, Lin Kong ${ }^{2,3}$, \\ Jiade J. Lu ${ }^{1,2}$ \\ ${ }^{1}$ Department of Radiation Oncology, Shanghai Proton and Heavy Ion Center, Shanghai, China; ${ }^{2}$ Shanghai Engineering Research Center of Proton \\ and Heavy Ion Radiation Therapy, Shanghai, China; ${ }^{3}$ Department of Radiation Oncology, Shanghai Proton and Heavy Ion Center, Fudan University \\ Shanghai Cancer Hospital, Shanghai, China \\ Contributions: (I) Conception and design: JJ Lu; (II) Administrative support: L Kong; (III) Provision of study patients: JJ Lu, L Kong; (IV) Collection \\ and assembly of data: J Yang, J Gao, J Hu, W Hu, X Qiu, Q Huang; (V) Data analysis and interpretation: J Yang; (VI) Manuscript writing: All \\ authors; (VII) Final approval of manuscript: All authors. \\ Correspondence to: Lin Kong; Jiade J. Lu. Shanghai Proton and Heavy Ion Center, 4365 Kangxin Road, Shanghai 201321, China. \\ Email: lin.kong@sphic.org.cn; jiade.lu@sphic.org.cn.
}

Background: Treatment of radiation-induced second primary malignancy (RI-SPM) is challenging and usually associated with poor outcomes. For patients with unresectable or incompletely resected diseases, carbon-ion radiotherapy (CIRT) offers physical and biologic advantages over photon-based re-irradiation. We report the results of salvage CIRT in 15 patients with RI-SPM.

Methods: Fifteen consecutive and non-selected patients with RI-SPM who underwent salvage CIRT at the Shanghai Proton and Heavy Ion Center between November 2015 and May 2019 were included in this retrospective study. CIRT doses were 57.5-69 Gy (RBE) [at 2.5-3.0 Gy (RBE)/daily fraction]. The actuarial 1-year overall survival (OS), locoregional progression-free survival (LPFS), distant metastasis-free survival (DMFS), and progression-free survival (PFS) rates as well as acute/late toxicities were analyzed.

Results: Among the 15 patients included, 10 were soft tissue sarcomas, 2 were chondrosarcomas, 1 was osteosarcoma, 1 was squamous cell carcinoma and 1 was esthesioneuroblastoma. With a median followup of 13.0 (range, 2.73-29.63) months, the actuarial 1-year OS, LPFS, DMFS, and PFS rates were 69.3\%, $53.0 \%, 92.9 \%$, and $48.2 \%$, respectively. No grade 2 and grade 3 acute adverse effect was observed. One patient experienced grade 4 hemorrhage which required embolization during CIRT, and lately died from hemorrhage (grade 5) at 3.4 months after the completion of CIRT. No other late adverse effects of $\geq$ grade 2 was observed.

Conclusions: Salvage CIRT provided relatively safe and effective short-term outcome for patients with unresectable or in-completely resected RI-SPM, as compared to historical data on re-irradiation using the conventional photon beam technology. However, further improvement in both disease control and toxicity prevention is needed.

Keywords: Carbon-ion radiotherapy (CIRT); radiation-induced malignancy; sarcoma

Submitted Jan 02, 2020. Accepted for publication Jan 09, 2020.

doi: $10.21037 /$ atm-20-200

View this article at: https://dx.doi.org/10.21037/atm-20-200 


\section{Introduction}

Radiation therapy (RT) is one of the key modalities for cancer treatment, and $\sim 70 \%$ of cancer patients will receive RT in their course of disease. With the significantly prolonged overall survival (OS) time resulted from the advances in the cancer treatment technology and strategy, treatment-induced late toxicity become a more substantial issue that perplexes patients and oncologists. Despite its effectiveness in cancer treatment, ionizing radiation is a known carcinogen. And radiation-induced second primary malignancy (RI-SPM) is one of the most devastating treatment-induced late toxicities torments patients.

RI-SPM was initially defined by Cohan as tumors that have arisen in an irradiated field with a different histology from the initial diagnosis, and after at least 5 years of latent period from the initial irradiation. In addition, the tissue in which the alleged SPMs arose must have been metabolically and genetically normal before the radiation exposure $(1,2)$. The incidence of RI-SPM ranges $0.7-0.9 \%$ for patients received RT and is commonly of mesenchymal nature (1). Despite its rarity, RI-SPM is usually highly malignant with aggressive biological behavior that needs aggressive surgical intervention. However, for patients whose disease initiated near critical organs, surgery with a negative margin may not be feasible. Re-irradiation for salvage treatment for unresectable or inoperable RI-SPM is challenging, as most malignancies of mesenchymal origin are usually radioresistant (3). Furthermore, the tolerance of the normal tissue adjacent to the RI-SPM usually limits the dose of a second course of radiotherapy at definitive dose.

Accelerated particle beam therapy is featured with distinct physical characteristics such as a low energy deposition within the entry path prior to the Bragg Peak formed by the steep dose deposition and a sharp dose fall-off thereafter, as well as a sharp lateral penumbra $(4,5)$. These characters enable precise and conformal dose distribution to the SPM foci thereby reduce the toxicities of nearby tissues heavily irradiated in the initial course of radiotherapy. Moreover, the higher relative-biological effectiveness (RBE) of heavy-ion (e.g., carbon ion) renders more effective killing for cells that are resistant to photon beam radiation (6). Both features are important for salvaging tumor adjacent to the regions that were heavily irradiated. The benefit of re-irradiation using carbon-ion radiotherapy (CIRT) in salvaging locally recurrent cancer has been repeatedly demonstrated (7-9). However, the theoretical benefits of CIRT in the salvage treatment of RI-SPM has not been sufficiently investigated. In the current study, we attempt to evaluate the outcomes in terms of survival, disease control, and adverse-effects of a relatively uniform group of patients with RI-SPM treated with CIRT re-irradiation.

\section{Methods}

This retrospective study was approved by the ethical board of SPHIC, the ID of this ethics approval is SPHICHNCNS-2017-03 (RS) and patient consent was waived. It was conducted in accordance with the Declaration of Helsinki (as revised in 2013).

\section{Characteristics of patients and their initial course of radiotherapy}

Between November 2015 and May 2019, 15 consecutive and non-selected patients with histologically confirmed RI-SPM were treated at the Shanghai Proton and Heavy Ion Center (SPHIC). The median age of the cohort was 54 (range, 32-62) years. Eleven patients were males and 4 were females. The most common sites of the disease included base of skull (6 patients), nasal-sinus (5 patients), and nasopharynx (3 patients). One patient who received adjuvant radiation for breast cancer developed SPM in the lower neck. Twelve (80\%) patients presented with locally advanced (T3/T4) disease, and 3 (20\%) had T1 (2 chondrosarcomas and 1 angiosarcoma) disease. No patient presented with distant metastasis $(\mathrm{DM})$ at the time of their diagnosis.

The median volume of tumors was 70.46 (range, $0-174.49) \mathrm{cm}^{3}$. One patient received adjuvant CIRT to the surgical bed for close surgical margin. Eleven patients received 50 to 75.4 Gy photon-based radiotherapy (at conventional fractionation) malignant pathologies, and 2 patients received stereotactic radiosurgery to 13 Gy to the $50 \%$ of the isodose line for pituitary adenoma. The radiation dose regimens of the remaining 2 patients were not provided. The median interval between the salvage CIRT and the initial course of radiation was 134 (range, 64-294) months.

The characteristics of patients, their disease and initial cancer diagnosis were detailed in Tables 1,2.

\section{Pretreatment evaluation and registration}

Evaluations before salvage CIRT included a complete history and physical (H\&P) examination, complete blood count (CBC), serum electrolytes, and hepatic/renal function 
Table 1 Characteristics of the patients, their disease, and prior treatment of the 15 cases with RI-SPM

\begin{tabular}{|c|c|c|c|c|c|c|c|c|}
\hline $\begin{array}{l}\text { Patient } \\
\text { No. }\end{array}$ & $\begin{array}{l}\text { Age } \\
\text { (year) }\end{array}$ & Gender* & Pathology & Site & $\mathrm{T}$ & $\mathrm{N}$ & $\begin{array}{c}\text { Tumor } \\
\text { volume/GTV }\left(\mathrm{cm}^{3}\right)\end{array}$ & $\begin{array}{l}\text { Previous } \\
\text { diagnosis }\end{array}$ \\
\hline 1 & 62 & 1 & Squamous cell carcinoma & Nasal-sinus & 3 & 0 & 174.49 & Hodgkin lymphoma \\
\hline 3 & 54 & 1 & Myofibroblastic sarcoma & Nasopharynx & 4 & 0 & 69.77 & NPC \\
\hline 6 & 61 & 1 & Spindle cell sarcoma & Base of skull & 4 & 0 & 94.85 & NPC \\
\hline 7 & 60 & 1 & Osteosarcoma & Nasal-sinus & 4 & 0 & 128.2 & NPC \\
\hline 8 & 51 & 1 & Spindle cell sarcoma & Base of skull & 4 & 0 & 70.46 & Pituitary adenoma \\
\hline 12 & 56 & 2 & Small round cell sarcoma & Base of skull & 4 & 0 & 38.05 & Pituitary adenoma \\
\hline 13 & 35 & 1 & Myofibroblastic osteosarcoma & Nasal-sinus & 4 & 0 & 77.28 & Myofibroblastic sarcoma \\
\hline 14 & 36 & 2 & Angiosarcoma & Neck & 1 & 0 & 0/no GTV, CTV 213.4 & Breast cancer \\
\hline 15 & 44 & 1 & Chondrosarcoma & Base of skull & 1 & 0 & 60.7 & Chordoma \\
\hline
\end{tabular}

*, 1 should be male, 2 should be female. RI-SPM, radiation-induced second primary malignancy; GTV, gross tumor volume; CTV, clinical target volume; NPC, nasopharyngeal carcinoma.

tests. As the lesions of all patients in this cohort located in the head and neck (including one breast cancer patient received adjuvant radiotherapy), MRI of the head and neck regions was required (CT was allowed when MRI was contraindicated). Positron emission tomography (PET)/ CT was recommended to all patients, but CT of the thorax, ultrasound or CT of the abdomen, and bone scan were acceptable if PET/CT was declined. Direct or fiberoptic endoscopy of the upper airway was performed when clinically indicated. The AJCC staging system (7th or 8th edition depended on the date of pathological diagnosis) was applied for all patients before any salvage treatment.

All cases were discussed in the multidisciplinary tumor (MDT) clinic of the SPHIC for their diagnoses, indications, and selection of particle radiotherapy protocol (IRB registered) prior to registration and immobilization. The data for diagnosis, initial treatment, and follow-ups were recorded to a prospective registry and database.

\section{Salvage carbon-ion RT}

All patients were immobilized in supine position with
AlphaCradle ${ }^{\circledR}$ and thermoplastic masks. CT scans of the affected region without intravenous contrast were performed for planning. MRI-CT fusion was performed for all patients prior to target volume delineation. The gross tumor volume (GTV) consisted of the gross tumor observed on clinical examination or imaging studies. We defined clinical target volume (CTV) as a GTV with a $1-3 \mathrm{~mm}$ margin to deliver the prescribed dose to the tumor. For patients who also received surgery and/or chemotherapy prior to salvage CIRT, the initial tumor bed prior to surgery/chemotherapy was defined as the CTV. A maximum of a $5 \mathrm{~mm}$ margin was typically added to the CTV for the planning target volume for uncertainty with regard to dose distribution and potential setup errors.

The initial radiotherapy plans were available and obtained from 13 patients and the doses to OARs were identified. Recovery from the dose of initial course of radiotherapy was set at $70 \%$ regardless of the latent time between the two courses of treatment (10).

Doses were measured by Gy (RBE) to account for the differences in biological effectiveness between carbon-ion and photon beams. The dose constraints of the optic nerve/ 


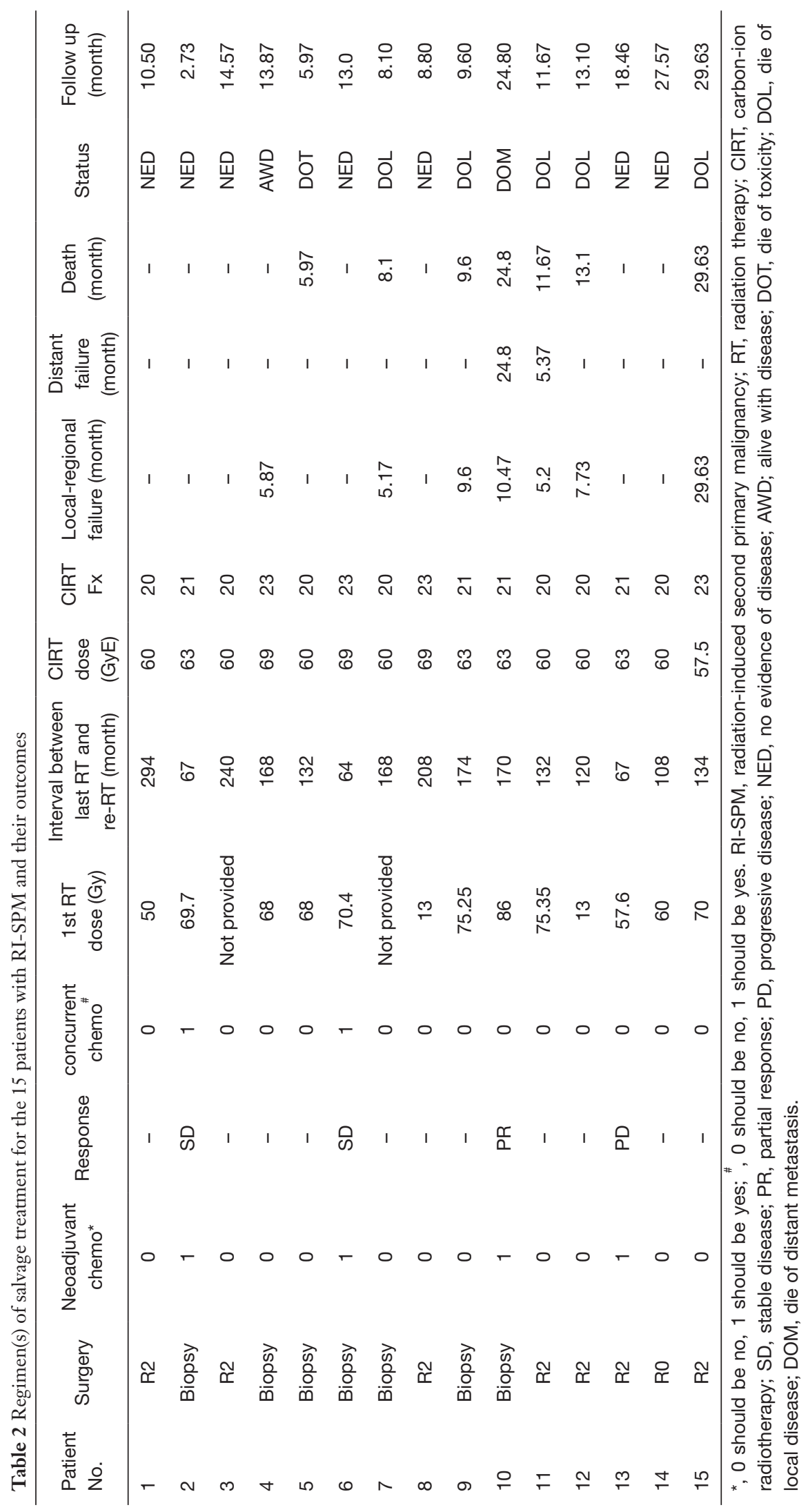


chiasm [ $\left.\mathrm{D}_{20}<30 \mathrm{~Gy}(\mathrm{RBE})\right]$, brain stem $\left[\mathrm{D}_{\max }<45 \mathrm{~Gy}(\mathrm{RBE})\right]$, temporal lobes $\left(\mathrm{V}_{40}<7.66 \mathrm{cc} ; \mathrm{V}_{50}<4.66 \mathrm{cc}\right)$, and spinal cord $\left[D_{\max }<30\right.$ Gy $\left.(\mathrm{RBE})\right]$, which were based on the protocol from the National Institute of Quantum and Radiation Science (NIQRS) of Japan (11). The dose constrains of all other organs at risk (OARs) were based on the TD5/5 described by Emami (12). Planning for intensity modulated CIRT using pencil beam scanning (PBS) technology was performed using the Syngo ${ }^{\circledR}$ treatment planning system (version VC11. Siemens, Erlangen). Typically, beam delivery from 2-3 directions (avoid any metal implant such as dental fillings) were used. Setup accuracy was verified with orthogonal $\mathrm{X}$-ray using bony landmarks as a reference. Weekly verification CT were required from the second week of CIRT to assess any changes in anatomy.

\section{Chemotherapy}

Neoadjuvant chemotherapy was used at the discretion of the medical oncologist. Concurrent chemotherapy with CIRT is not recommended at SPHIC unless otherwise indicated.

\section{Follow-up}

All patients were required to comply with the standardized follow-up protocol of SPHIC. The first follow-up was scheduled between 4-6 weeks after the completion of CIRT. Patients were then required to be followed up every 3 months in the first 2 years, every 6 months in the following 3 years, and annually thereafter. A complete H\&P examination and imaging studies (MRI preferred) focused on the region(s) of the SPM are required at each followup session. CT of the thorax, CT or ultrasound of the abdomen, PET-CT and other laboratory or imaging studies were ordered if clinically indicated.

\section{Data analysis}

Duration of survival was calculated from the diagnosis of the second primary tumor until death or the date of the last follow-up. Time to local, regional, and/or distant failure or progression was measured from the date of any treatment for RI-SPM until documented treatment failure. Rates of survivals were calculated using the Kaplan-Meier method (13). All analyses were performed in SPSS statistics version 18.0 software package (Chicago, IL, USA).

Acute and late adverse events were scored according to Radiation Therapy Oncology Group (RTOG) acute and late radiation morbidity scoring systems. Acute toxicities included events that occurred during or within 3 months after the initiation of salvage CIRT. Late toxicities covered those observed beginning at or persisted $>90$ days after completion of CIRT.

\section{Results}

\section{Treatment}

Seven patients underwent biopsy, 7 underwent R2 resection, and 1 achieved complete (R0) resection with close margin. Neoadjuvant chemotherapy was provided to 4 patients. One and 3 patients achieved partial response (PR) and stable disease (SD), respectively. Concurrent chemotherapy was applied for 2 patients at the discretion of the medical oncologists. The details of treatment of each patient prior to CIRT were detailed in Table 2.

All patients completed planned CIRT without unplanned break. The details of the dose regimens of salvage CIRT used were detailed in Table 2. Seven, 4, and 3 patients presented with gross disease were treated to 60, 63, and 69 Gy (RBE), respectively, at 3 Gy (RBE) per daily fraction. The patient who achieved $\mathrm{R} 0$ resection received $60 \mathrm{~Gy}$ (RBE) in 20 daily fractions. One patient with low grade chondrosarcoma received 57.5 Gy (RBE) in 23 fractions.

\section{Disease control and survival}

With a median follow-up of 13.0 (range, 2.73-29.63) months, local failure presented in 7 patients, mostly occurred within 1 year after the completion of CIRT ( 6 cases). DM occurred in 2 patients at 5.37 and 24.8 months after CIRT, respectively. Among these patients, 2 had both local and distant failure. Eight patients were alive including 7 cases with no evidence of disease (NED) and 1 alive with disease (AWD). Seven patients had died: 5 succumbed of local recurrence or progression (died of local disease, DOL), 1 died of distant metastasis (DOM), and another died of toxicity (DOT). The actuarial 12-month OS, local-regional relapse-free survival (LRFS), distant metastasis-free survival (DMFS), and progression-free survival (PFS) were $69.3 \%, 53.0 \%, 92.9 \%$ and $48.2 \%$, respectively (Figure 1 ).

The details of disease control and survival of each patient were displayed in Table 2.

\section{Adverse effects}

Two, 2, and 1 patients experienced grade 1 oral mucositis, 

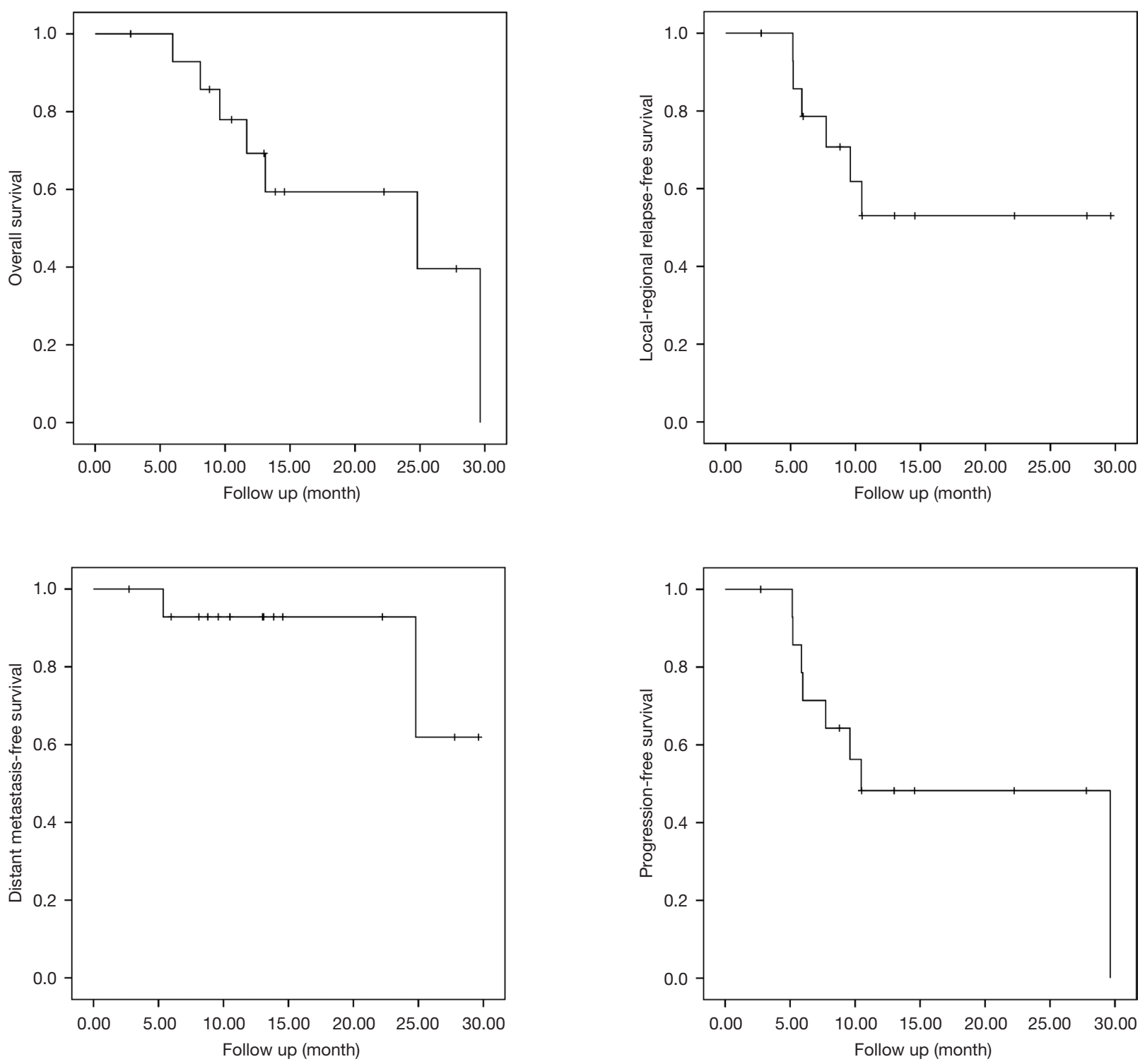

Figure 1 The OS, LRFS, DMFS and PFS of 15 RI-SPM patients treated by salvage CIRT. OS, overall survival; LRFS, locoregional progression-free survival; DMFS, distant metastasis-free survival; PFS, progression-free survival; RI-SPM, radiation-induced second primary malignancy; CIRT, carbon-ion radiotherapy.

grade 1 dermatitis, and grade 1 hemorrhage of nasal vestibule, respectively. One patient with pleomorphic sarcoma induced by IMRT (68 Gy in 30 fractions) for NPC experienced grade 4 acute hemorrhage from the sphenopalatine artery during CIRT. The bleeding was salvaged by embolism. The patient received resection of the tumor in the post-nasal space 1 month before the initiation of salvage CIRT, then completed to $60 \mathrm{~Gy}$ (RBE) in 20 fractions. As the bleeding sphenopalatine artery was encompassed in both the initial and salvage radiation fields, this adverse event of grade 4 hemorrhage was defined to be radiation-induced. The same patient later died from severe (grade 5) hemorrhage 3.5 months after the completion of salvage CIRT. The exact site of the bleeding that caused death was not confirmed. The maximum and mean doses from the salvage CIRT to the left/right carotid arteries were 61.21/61.22 Gy (RBE) and 56.52/51.86 Gy (RBE) (in 20 fractions). Unfortunately, the doses to the carotid arteries from the initial IMRT were not available to us, although it was likely that the full dose of 68 Gy from the initial 
Table 3 Type and frequency of acute adverse events

\begin{tabular}{lcccc}
\hline \multirow{2}{*}{ Toxicity } & \multicolumn{3}{c}{ Grade } \\
\cline { 2 - 5 } & 1, No. $(\%)$ & 2, No. (\%) & 3, No. (\%) & 4, No. (\%) \\
\hline Mucous membrane & $2(13.3)$ & $0(0.0)$ & $0(0.0)$ & $0(0.0)$ \\
Skin & $2(13.3)$ & $0(0.0)$ & $0(0.0)$ & $1(6.6)$ \\
Haemorrhage & $1(6.7)$ & $0(0.0)$ & $0.0)$ \\
\hline
\end{tabular}

Table 4 Type and frequency of late adverse events

\begin{tabular}{|c|c|c|c|c|c|}
\hline Toxicity & \multicolumn{5}{|c|}{ Grade } \\
\hline Dry mouth & $3(20.0)$ & $0(0.0)$ & $0(0.0)$ & $0(0.0)$ & - \\
\hline Skin & $1(6.7)$ & $0(0.0)$ & $0(0.0)$ & $0(0.0)$ & $0(0.0)$ \\
\hline Hemorrhage & $0(0.0)$ & $0(0.0)$ & $0(0.0)$ & $0(0.0)$ & $3(20.0)$ \\
\hline
\end{tabular}

IMRT covered both arteries for the locally advanced NPC. Nevertheless, bleeding from a carotid blowout could not be ruled out and was likely the cause of his fatal hemorrhage. No late adverse effects of grade 2-4 was observed otherwise. Grade 1 dry mouth, dermatitis, hearing impairment were observed in 3,1 , and 1 patient, respectively.

The types and severity of acute and late adverse events are detailed in Tables 3,4.

\section{Discussion}

RI-SPM is a rare but devastating treatment-associated late complication. Outcomes of patients with unresectable or inoperable RI-SPM are universally dismal, as most RI-SPM are of mesenchymal origin and usually resistant to photonbased radiotherapy and chemotherapy. In addition, dose of salvage re-irradiation is usually limited by the tolerance of OARs adjacent to the SPM foci. In this retrospective series, we analyzed the treatment outcomes of 15 patients treated with a relatively uniform fashion using CIRT for their RISPM. Twelve of the 15 patients (80\%) were diagnosed with secondary bone or soft-tissue sarcoma. All but one patient presented with gross disease, and the majority (12/15) had locally advanced disease. With a median follow-up of 13 months, the 1-year OS and locoregional progression-free survival (LPFS) rates were at $69.3 \%$ and $53.0 \%$. Although severe acute toxicity was observed in only one patient (grade 4 hemorrhage), 3 patients died of hemorrhage with or without evidence of disease progression.

Modern photon-based radiotherapy technologies including IMRT and stereotactic body radiotherapy (SBRT) have been used for the treatment of RI-SPM. However, it is very difficult to compare our outcomes of RI-SPM after CIRT re-irradiation with those after IMRT or SBRT for several reasons. First, data on disease control and patients' survival after IMRT or SBRT re-irradiation for SPM were often reported with those for local recurrence (14-16). Second, more patients in the historical data received salvage surgery before IMRT/SBRT. Salvage surgery prior to reirradiation with IMRT/SBRT was reported as a significant prognostic factor for improved OS in several studies $(15,16)$. On the contrary, only 1 in our cohort achieved meaningful surgery (R0 resection but with close margin). Nevertheless, the results of a multi-institutional in silico study of 25 cases for re-irradiation demonstrated clear dosimetric benefits from intensity-modulated proton or ion therapy, as compared to IMRT. Furthermore, the 1-year OS of close to $70 \%$ appeared to be substantially superior than 50 $58.4 \%$ after IMRT/SBRT re-irradiation for head and neck recurrence or SPM without salvage surgery $(15,16)$.

The results of salvage proton therapy for SPM were also reported with locoregional recurrence for head and neck cancers due to the extremely limited number of patients with SPM. Only 6 of the 61 patients presented with SPM in a study on the use of proton therapy for recurrent or secondary head and neck cancers published 
in 2016 (17). The 1- and 2-year OS rates were 57\% and $32.7 \%$, respectively, in the above-mentioned report. Not surprisingly, the characteristics of the patients and their diseases (such as pathology and stage at diagnoses of the SPMs) were not detailed. In a prospective study of proton reirradiation for recurrent and secondary soft tissue sarcoma (18), Guttmann et al. reported the results of 23 patients including 6 with SPM. Approximately half of the cohort presented with disease in the limb, and 7 had pelvic or retroperitoneal sarcomas, and only 2 patients presented with head and neck recurrence. In addition, $11(\sim 48 \%)$ patients achieved R1 or R0 resection prior to proton therapy. The authors reported a 1 - and 3-year OS of $100 \%$ and $64 \%$, respectively, but 12 patients experienced local failure at a median of 10 months. No grade $4 / 5$ and only one case of grade 3 toxicity was observed. Again, a meaningful comparison between the outcomes of our cohort and those after proton therapy re-irradiation is not feasible due to the difference in the nature of the disease, i.e., RISPM vs. mostly local recurrence after irradiation. However, the probability of treatment-associated severe toxicity, whether acute or late, appeared to be similar between our results and those after proton therapy. As far as we know, no results of salvage CIRT for RI-SPM has been reported, except for one on pulmonary re-irradiation for primary or metastatic cancers including one case of secondary tumor after radiotherapy for thymoma, as well as those reported from our institute $(19,20)$.

One key strength of the current study was the relatively uniform characteristics of patients as well as the uniform fashion of salvage radiation technique used for all cases. As far as we know, we are reporting the first clinical series of RI-SPM treated with salvage PBRT. However, our study has several limitations including its retrospective nature, relatively small sample size and short follow-up time. All patients except for one received initial radiotherapy for malignancies of the head and neck region, including 7 with nasopharyngeal cancer. One patient treated for SPM after adjuvant radiotherapy for breast cancer. As such, although our investigation is limited to only 15 patients, it represents the largest series of head and neck RI-SPM salvaged with modern radiation technology. However, whether the outcomes can be referenced for RI-SPM of other anatomical regions is subject to further investigation. Two patients could not provide the details of their initial course of radiation due to the extended latent period (168 and 294 months, respectively). One of the 2 patients developed local failure after 5 months and succumbed from disease progression. Both patients received $60 \mathrm{~Gy}$ (RBE) in 20 daily fractions, which is lower than the standard dose regimen [63 Gy (RBE) in 21 fractions] of our institutional treatment protocol due to the concern of toxicity. Fortunately, no severe toxicity was observed from both patients, but whether further dose escalation could improve disease control is unknown.

Only 2 of the 13 patients in our study developed DM after salvage CIRT. Both patients experienced local recurrence prior to DM. Local recurrence, which occurred in 7 patients including the 2 in concurrent with DM, appeared to be the most common cause of treatment failure and death. Clearly, further investigation will be needed to understand whether higher CIRT dose can improve local disease control without severe toxicities. The use of systemic treatment in concurrent with CIRT is another subject of investigation. In view of low responsiveness of sarcomas to chemotherapy, future direction of research on combined treatment may consider molecular targeted and immuno-therapy with PBRT.

\section{Conclusions}

RI-SPM is a rare but devastating late toxicity from radiotherapy. CIRT provided a 1 -year OS rate of close to $70 \%$ for patients mostly with locally advanced and unresectable RI-SPM. Fatal hemorrhage not associated with disease progression was observed in only 1 patient during and after the completion of CIRT and was the only severe late toxicity observed. Disease control needed improvement as LPFS approximated $50 \%$ at one year after salvage CIRT. Further investigation with the combined use of high-dose salvage CIRT and systemic treatment is needed.

\section{Acknowledgments}

Funding: This study was supported by grants from the Scientific Research projects of the Shanghai Science and Technology Committee (Project No. 19411951000), Shanghai Municipal Commission of Health and Family Planning (Project No. 20174Y0076), and Pudong New Area Science and Technology Development Foundation (Project No. PKJ2018-Y50).

\section{Footnote}

Conflicts of Interest: All authors have completed the ICMJE uniform disclosure form (available at https://atm. 
amegroups.com/article/view/10.21037/atm-20-200/coif). The authors have no conflicts of interest to declare.

Ethical Statement: The authors are accountable for all aspects of the work in ensuring that questions related to the accuracy or integrity of any part of the work are appropriately investigated and resolved. This retrospective study was approved by the ethical board of SPHIC, the ID of this ethics approval is SPHIC-HNCNS-2017-03 (RS) and patient consent was waived. It was conducted in accordance with the Declaration of Helsinki (as revised in 2013).

Open Access Statement: This is an Open Access article distributed in accordance with the Creative Commons Attribution-NonCommercial-NoDerivs 4.0 International License (CC BY-NC-ND 4.0), which permits the noncommercial replication and distribution of the article with the strict proviso that no changes or edits are made and the original work is properly cited (including links to both the formal publication through the relevant DOI and the license). See: https://creativecommons.org/licenses/by-nc-nd/4.0/.

\section{References}

1. Kong L, Lu JJ. Second Primary Tumors. In: Schwab Meds in Encyclopedia of Cancer. Springer, 2014.

2. Cahan WG, Woodard HQ. Sarcoma arising in irradiated bone; report of 11 cases. Cancer 1948;1:3-29.

3. Kepka L, DeLaney TF, Suit HD, et al. Results of radiation therapy for unresected soft-tissue sarcomas. Int J Radiat Oncol Biol Phys 2005;63:852-9.

4. Kanai T, Endo M, Minohara S, Miyahara N, et al. Biophysical characteristics of HIMAC clinical irradiation system for heavy-ion radiation therapy. Int J Radiat Oncol Biol Phys 1999;44:201-10.

5. Tsujii H, Kamada T, Baba M. Clinical advantages of carbon-ion radiotherapy. New J Phys 2008;10.

6. Durante M, Orecchia R, Loeffler JS. Charged-particle therapy in cancer: clinical uses and future perspectives. Nat Rev Clin Oncol 2017;14:483-95.

7. $\mathrm{Hu}$ J, Bao C, Gao J, et al. Salvage treatment using carbon ion radiation in patients with locoregionally recurrent nasopharyngeal carcinoma: Initial results. Cancer 2018;124:2427-37.

8. Romesser PB, Cahlon O, Scher ED, et al. Proton Beam Reirradiation for Recurrent Head and Neck Cancer: Multi-institutional Report on Feasibility and Early Outcomes. Int J Radiat Oncol Biol Phys 2016;95:386-95.
9. Gao J, Hu J, Guan X, et al. Salvage Carbon-Ion Radiation Therapy For Locoregionally Recurrent Head and Neck Malignancies. Sci Rep 2019;9:4259.

10. Nieder C, Milas L, Ang KK. Tissue tolerance to reirradiation. Semin Radiat Oncol 2000;10:200-9.

11. Koto M. Skull base and upper cervical spine tumors. In: Tsujii H, Kamada T, Shirai T. editors. CarbonIon Radiotherapy Principles, Practices, and Treatment Planning. Heidelberg: Springer; 2014.

12. Emami B, Lyman J, Brown A, et al. Tolerance of normal tissue to therapeutic irradiation. Int J Radiat Oncol Biol Phys 1991;21:109-22.

13. Dinse GE, Lagakos SW. Nonparametric estimation of lifetime and disease onset distributions from incomplete observations. Biometrics 1982;38:921-32.

14. Choi SH, Chang JS, Choi J, et al. Re-irradiation Using Intensity-modulated Radiotherapy for Recurrent and Second Primary Head and Neck Cancer. Anticancer Res 2018;38:3165-73.

15. Vargo JA, Ward MC, Caudell JJ, et al. A Multiinstitutional Comparison of SBRT and IMRT for Definitive Reirradiation of Recurrent or Second Primary Head and Neck Cancer. Int J Radiat Oncol Biol Phys 2018;100:595-605.

16. Velez MA, Veruttipong D, Wang PC, et al. Re-irradiation for recurrent and second primary cancers of the head and neck. Oral Oncol 2017;67:46-51.

17. McDonald MW, Zolali-Meybodi O, Lehnert SJ, et al. Reirradiation of Recurrent and Second Primary Head and Neck Cancer With Proton Therapy. Int J Radiat Oncol Biol Phys 2016;96:808-19.

18. Guttmann DM, Frick MA, Carmona R, et al. A prospective study of proton reirradiation for recurrent and secondary soft tissue sarcoma. Radiother Oncol 2017;124:271-6.

19. Yang J, Gao J, Wu X, et al. Salvage Carbon Ion Radiation Therapy for Locally Recurrent or Radiation-Induced Second Primary Sarcoma of the Head and Neck. J Cancer 2018;9:2215-23.

20. Hayashi K, Yamamoto N, Karube M, et al. Feasibility of carbon-ion radiotherapy for re-irradiation of locoregionally recurrent, metastatic, or secondary lung tumors. Cancer Sci 2018;109:1562-9.

Cite this article as: Yang J, Gao J, Hu J, Hu W, Qiu X, Huang Q, Kong L, Lu JJ. Carbon-ion radiotherapy in the treatment of radiation-induced second primary malignancies. Ann Transl Med 2022;10(22):1200. doi: 10.21037/atm-20-200 